\title{
On homology of associative shelves
}

\author{
Alissa S. Crans ${ }^{1}$ • Sujoy Mukherjee ${ }^{2}$. \\ Józef H. Przytycki ${ }^{2,3}$
}

Received: 1 April 2016 / Accepted: 15 November 2016 / Published online: 19 December 2016 (C) Tbilisi Centre for Mathematical Sciences 2016

\begin{abstract}
Homology theories for associative algebraic structures are well established and have been studied for a long time. More recently, homology theories for selfdistributive algebraic structures motivated by knot theory, such as quandles and their relatives, have been developed and investigated. In this paper, we study associative self-distributive algebraic structures and their one-term and two-term (rack) homology groups.
\end{abstract}

Keywords Spindles · Knot theory - One-term and two-term (rack) distributive homology $\cdot$ Unital $\cdot$ Self-distributive semigroups $\cdot$ Laver tables

Communicated by Vladimir Vershinin.

Alissa S. Crans was supported by a grant from the Simons Foundation (\#360097, Alissa Crans). Sujoy Mukherjee was supported by the Presidential Merit Fellowship of the George Washington University. Józef H. Przytycki was partially supported by the Simons Foundation Collaboration Grant for Mathematicians-316446.

$凶 \quad$ Józef H. Przytycki

przytyck@gwu.edu

Alissa S. Crans

acrans@1mu.edu

Sujoy Mukherjee

sujoymukherjee@gwu.edu

1 Department of Mathematics, Loyola Marymount University, Los Angeles, CA, USA

2 Department of Mathematics, The George Washington University, Washington DC, USA

3 University of Gdańsk, Gdańsk, Poland 
Mathematics Subject Classification Primary 18G60; Secondary 20M32, 20N02, $57 \mathrm{M} 25$

Self-distributive structures ${ }^{1}$ have been studied for over a century. In 1880 Peirce [20] emphasized the importance of right self-distributivity in algebraic structures, when his friend Schröder [27] constructed an example of a non-associative three element magma. Two-sided self-distributive structures were studied in 1929 by Burstin and Mayer [2]; compare [23]. In the past several decades, self-distributive structures have secured an important role in knot theory.

In May of 2015, during a discussion of the origin of the word 'quandle' on the $n$-Category Café (https://golem.ph.utexas.edu/category/), Sam C. ${ }^{2}$ remarked that unital shelves are associative and provided a short elegant proof. The work herein was inspired by this post. In Sect. 1, we provide definitions and discuss some elementary propositions. The first two of these propositions were proven by Sam C. [26]. The latter propositions lay the pathway for the subsequent sections.

Section 2 addresses finitely generated associative shelves, their cardinalities and generating functions. Following this, finitely generated proto-unital shelves, pre-unital shelves, and unital shelves are discussed. While some of the observations in this section are new, others are stated for completeness [11,12,29].

In Sect. 3, the two well-known homology theories for self-distributive algebraic structures, namely one-term distributive homology and two-term (rack) homology are defined and the main results concerning the homology groups of a special class of associative shelves are proven. In particular, we compute one-term and two-term (rack) homology of unital shelves. The main theorem concerning two-term homology of shelves with right fixed elements extends to the two-term (rack) homology groups of Laver tables $[4,5,16]$ and f-block spindles [3,24]. Henceforth, we will use the term two-term homology to refer to rack homology.

Finally, Sect. 4 and the Appendix contain conjectures and computational data.

\section{Introduction}

Definition 1.1 A quandle $(X, *)$ is a magma ${ }^{3}$ satisfying the following properties:

(1) (idempotence) $a * a=a$ for all $a \in X$,

(2) (inverse) There exists $\bar{*}: X \times X \longrightarrow X$ such that $(a \bar{*} b) * b=a=(a * b) \bar{*} b$ for all $a, b \in X$, and

(3) (self-distributivity) $(a * b) * c=(a * c) *(b * c)$ for all $a, b, c \in X$

The three properties satisfied by the elements of a quandle are the algebraic analogues of the conditions satisfied by quandle colorings under the three Reidemeister moves, depicted in Fig. 1. The convention for coloring arcs of a knot diagram by elements of a quandle $X$ is illustrated in Figs. 2 and 3. The primordial example of a

\footnotetext{
1 The word distributivity was coined in 1814 by French mathematician François Servois (1767-1847).

2 Unfortunately we do not know his full name. In [26], he writes: "I am not a mathematician or physicist...".

3 The term 'magma' was introduced by Jean-Pierre Serre in 1965. The older term 'groupoid,' introduced by Øystein Ore in 1937, now has different meaning: it is a category in which every morphism is invertible.
} 
Fig. 1 Unoriented Reidemeister moves; coherent orientations could be added
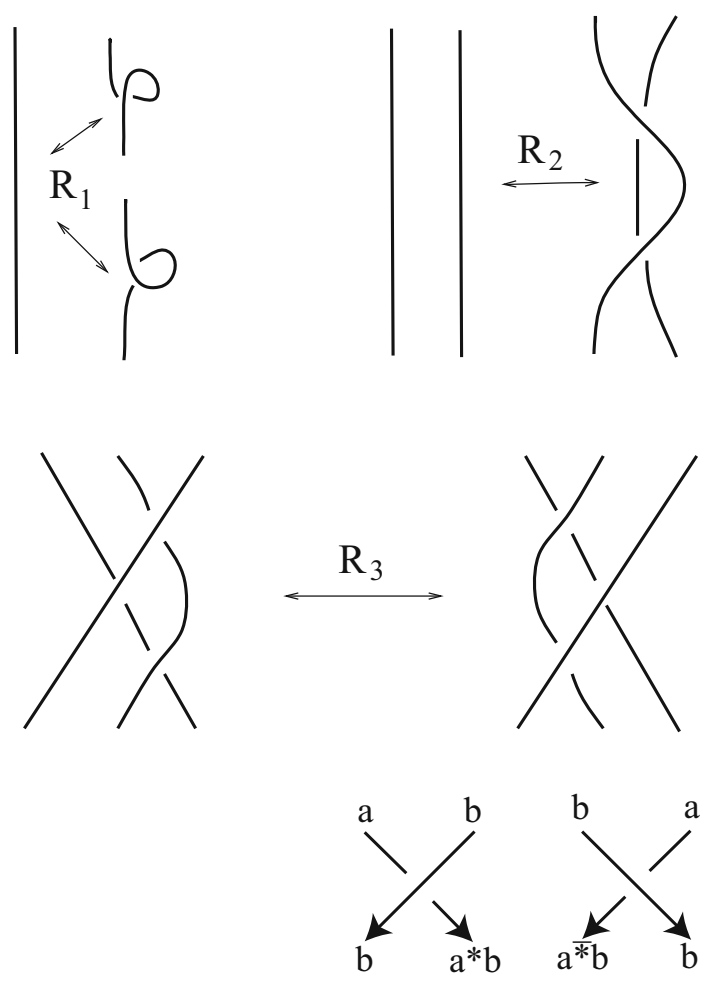

Fig. 2 Quandle coloring rules

Fig. 3 Reidemeister move 3 and quandle coloring
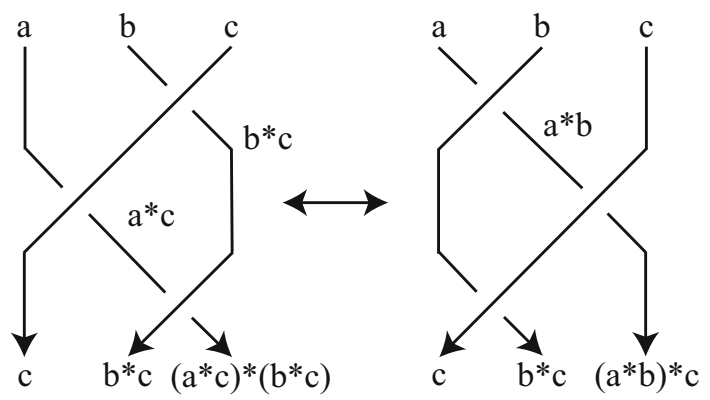

quandle arises when starting with a group $(G, \cdot)$ and defining the quandle operation as $a * b=b^{-1} \cdot a \cdot b$ for all $a, b \in G$.

Definition 1.2 By relaxing some of the axioms of a quandle, we obtain generalizations of quandles, including:

(1) A rack is a magma which satisfies the second and third quandle axioms.

(2) A spindle is a magma which satisfies the first and the third axioms of a quandle.

(3) A shelf is a magma which satisfies only the third quandle axiom.

(4) A unital shelf is a shelf $(X, *)$ equipped with an element 1 , such that $x * 1=$ $x=1 * x$ for all $x \in X$. 
Table 1 An associative spindle

\begin{tabular}{l|llll}
$*$ & $a$ & $b$ & $c$ & $d$ \\
\hline$a$ & $a$ & $a$ & $c$ & $d$ \\
$b$ & $a$ & $b$ & $c$ & $d$ \\
$c$ & $a$ & $a$ & $c$ & $d$ \\
$d$ & $a$ & $d$ & $c$ & $d$
\end{tabular}

Table 1 depicts an associative spindle that is not a quandle. We continue with some basic observations, due to Sam C.

Proposition 1.3 (Sam C.) Let $(X, *)$ be a unital shelf. Then:

(0) $a * a=a$ for all $a \in X$

(1) $a * b=b *(a * b)$ for all $a, b \in X$

(2) $a * b=(a * b) * b$ for all $a, b \in X$

Proof Let $a, b \in X$, where $X$ is a unital shelf. Then:

(0) $a=(1 * 1) * a=(1 * a) *(1 * a)=a * a$

(1) $a * b=(1 * a) * b=(1 * b) *(a * b)=b *(a * b)$

(2) $a * b=(a * 1) * b=(a * b) *(1 * b)=(a * b) * b$

Remark 1.4 (1) Monoids satisfying condition (1) of Proposition 1.3 are known as 'graphic monoids' and have been studied in $[13,28]{ }^{4}$

(2) Semi-groups satisfying $b *(a * b)=a * b$ are called graphic semi-groups. It turns out graphic semi-groups are shelves because $(a * c) *(b * c)=a *(c *(b * c))=$ $a *(b * c)=(a * b) * c$. In particular, graphic monoids are self-distributive so that unital shelves and graphic monoids are the same algebraic structures.

(3) In Proposition 1.3, the proofs of parts (0) and (1) require only the left-sided unity, whereas for the proof of part (2), both the left-sided and right-sided units are needed.

Definition 1.5 A shelf satisfying axioms (0), (1), and (2) of Proposition 1.3 is called a pre-unital shelf and a shelf satisfying properties (1) and (2) of Proposition 1.3 is called a proto-unital shelf.

Corollary 1.6 (Sam C.) Proto-unital shelves are associative. In particular, this implies unital shelves are associative.

Proof Let $a, b, c \in X$, where $X$ is a proto-unital shelf. Then:

$$
\begin{aligned}
(a * b) * c & =(a * c) *(b * c)=(a *(b * c)) *(c *(b * c))=(a *(b * c)) *(b * c) \\
& =a *(b * c)
\end{aligned}
$$

\footnotetext{
4 In [13], the number of elements in the free graphic monoid was computed. The term graphic monoid was coined by Lawvere [15].
} 
Table 2 A non-associative quasi-group quandle

\begin{tabular}{l|llll}
$*$ & $a$ & $b$ & $c$ & $d$ \\
\hline$a$ & $a$ & $c$ & $d$ & $b$ \\
$b$ & $d$ & $b$ & $a$ & $c$ \\
$c$ & $b$ & $d$ & $c$ & $a$ \\
$d$ & $c$ & $a$ & $b$ & $d$
\end{tabular}

We conclude by illustrating a relationship between pre-unital and unital shelves.

Proposition 1.7 If $(X, *)$ is a pre-unital shelf, then $(X \cup\{1\}, *)$ is unital. Conversely, if $(X, *)$ is a unital shelf, then $(X \backslash\{1\}, *)$ is pre-unital.

Proof Extending a pre-unital shelf to a unital shelf requires verifying the selfdistributivity property for all $a, b, c \in X$ where at least one of these elements is the unit. This follows easily. Conversely, for two elements $a, b \in X$, neither of which is the unit, $a * b \neq 1$. Namely, if $a * b=1$ then $(a * b) * b=b$. Thus by condition (2) of Proposition 1.3, $a * b=b$, and $b=1$. Therefore, the result follows.

In the sections that follow, we study various properties of associative shelves, but not of associative racks and quandles, even though these two algebraic structures are more important from a knot-theoretic point of view. This is due to the fact that associative racks are trivial. If $(X, *)$ is an associative rack with $a, b \in X$, then $(a * b) * b=(a * b) *(b * b)$. But this implies, $(((a * b) * b) * b) * b=$ $(((a * b) *(b * b)) \bar{*}) \bar{*} b$. As $(X, *)$ is associative, we may ignore the parentheses. Hence, it follows that $a=a * b$.

Definition 1.8 A quasi-group $(X, *)$ is a magma satisfying:

(1) The equation $a * x=b$ has a unique solution in $X$ for all $a, b \in X$

(2) The equation $x * a=b$ has a unique solution in $X$ for all $a, b \in X$

Finite quasi-groups are also known as 'Latin squares' and were introduced by Euler in the late 1700s. The two axioms of a Latin square $X$ of order $n$ are equivalent to the condition that the rows and columns of the multiplication table are permutations of the $n$ elements in $X$. Quandles, by definition satisfy the second axiom of Definition 1.8. If they also satisfy the first axiom of Definition 1.8 they are called quasi-group quandles. Table 2 illustrates a non-associative quasi-group quandle.

Proposition 1.9 Let $(X, *)$ be a quasi-group satisfying $(a * b) * b=a * b$ (condition (2) of Proposition 1.3). Then, $X$ has only one element.

Proof Let $a, b \in X$. We have $(a * b) * b=a * b$ which implies $a * b=a$ by the second axiom of quasi-groups; in particular $a * a=a$. Now by the first axiom of quasi-groups, we have $a * a=a$ and $a * b=a$, so that $a=b$, and thus there is only one element in $X$.

Using Microsoft Visual C++, preliminary computations produced the data in Table 3 regarding the number of associative shelves (AS), associative spindles (ASp), 
Table 3 Preliminary computations

\begin{tabular}{|c|c|c|c|}
\hline$n$ & $\# A S$ & $\# A S p$ & $\# U S$ \\
\hline 1 & 1 & 1 & 1 \\
\hline 2 & 4 & 3 & 1 \\
\hline 3 & 16 & 9 & 4 \\
\hline 4 & 93 & 38 & 6 \\
\hline
\end{tabular}

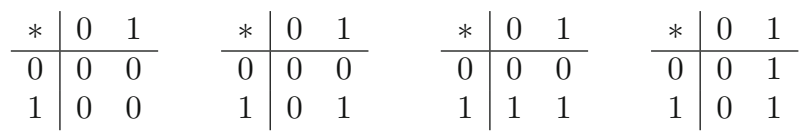

Fig. 4 The four associative shelves of order two up to isomorphism

and unital shelves (US) of up to four elements. However, much more has been computed for semi-groups satisfying different combinations of axioms [11]. Up to isomorphism, there are four associative shelves of order two, which are depicted in Fig. 4. All but the first are spindles.

\section{Finitely generated associative shelves}

In this section, we consider finitely generated objects and prove that they are finite. Moreover, for these objects, we find normal forms, count number of elements and provide generating functions.

We will denote our alphabet by $A$, the monoid of words in alphabet $A$ with juxtaposition as the operation (denoted by $*^{5}$ ) by $A^{\star}$, and the unit element of the monoid, namely the empty word, by $\emptyset$. If we delete the empty word we obtain the semi-group $A^{\star} \backslash\{\emptyset\}$.

Definition 2.1 Let $\sim$ be an equivalence relation defined on the words of $A^{\star} \backslash\{\emptyset\}$ by:

(1) Two words in $A^{\star} \backslash\{\emptyset\}$ are equivalent under $\sim$ if one can be transformed into the other by a finite number of right self-distributive operations with respect to juxtaposition. In other words, $\sim$ is the smallest equivalence relation in $A^{\star} \backslash\{\emptyset\}$ generated by $a * b * c=a * c * b * c$, where $a, b, c \in A^{\star} \backslash\{\emptyset\}$ (compare [21]). The free associative shelf on $A$, denoted by $F A S(A)$, is given by:

$$
F A S(A)=\frac{A^{\star} \backslash\{\emptyset\}}{\sim}
$$

(2) Two words in $A^{\star} \backslash\{\emptyset\}$ are equivalent under $\sim$ if one can be transformed into the other using a finite number of the axioms of proto-unital shelves with respect to juxtaposition. The free proto-unital shelf on $A$, denoted by $F P U S(A)$, is given

5 In the rest of the section, to simplify notation we often omit $*$. 
by:

$$
F P U S(A)=\frac{A^{\star} \backslash\{\emptyset\}}{\sim}
$$

(3) Two words in $A^{\star} \backslash\{\emptyset\}$ are equivalent under $\sim$ if one can be transformed to the other using a finite number of the axioms of pre-unital shelves with respect to juxtaposition. The free pre-unital shelf on $A$, denoted by $F \widetilde{P} U S(A)$, is given by:

$$
F \widetilde{P} U S(A)=\frac{A^{\star} \backslash\{\emptyset\}}{\sim}
$$

$F A S(A)$ can equivalently be defined by the following universal property. If $f$ : $A \longrightarrow Y$ is a map between sets where $Y$ is an associative shelf, then $f$ can be uniquely extended to the homomorphism $\widetilde{f}: F A S(A) \longrightarrow Y$. Similarly, FPUS $(A)$ and $F \widetilde{P} U S(A)$ can equivalently be defined using universal properties. If $A$ is finite with $n$ elements, we denote $F A S(A), F P U S(A)$ and $F \widetilde{P} U S(A)$ by $F A S(n), F P U S(n)$ and $F \widetilde{P} U S(n)$, respectively.

\subsection{Free associative shelves}

The structure of $F A S(n)$ was studied by a Czech group from Prague. The extensive survey of their work can be found in [11].

Note that it is enough to define ' $\sim$ ' on the generators $A_{n}$ of $F A S(n)$. This can be proven by induction on the length of the words by considering three arbitrary words.

Intuition suggests that to obtain a word in its reduced form, it is enough to consider reductive self-distributive operations. However, this is not often the case.

We can reduce any word in $A_{n}^{\star} \backslash\{\emptyset\}$ uniquely to the normal form via the process described below. The crucial observation is that doing so requires the relation $a b^{2} c \sim$ $a b c$, described in the following lemma.

Lemma 2.2 Let $a, b, c \in F A S(n)$. Then $a b^{2} c=a b c$.

Proof

$$
\begin{aligned}
a b b c & =a((b b) c)=a((b c)(b c))=((a b)(c b)) c=((a c) b) c=(a c)(b c) \\
& =(a b) c=a b c
\end{aligned}
$$

The above equations illustrate how associativity and right self-distributivity go hand in hand. For small values of $n$, computations produce:

$$
F A S(1)=\left\{a, a^{2}, a^{3}\right\}, \text { and }
$$

$F A S(2)=\left\{a, b, a b, b a, a^{2}, b^{2}, b^{3}, a b^{2}, b a b, b^{2} a, a^{2} b, a b a, b a^{2}, a^{3}, b a b^{2}, a b a^{2}, a^{2} b^{2}, b^{2} a^{2}\right\}$

Table 4 displays the multiplication table of $F A S(2)$ where we have replaced the above words with integers from 0 to 17 in the order above. Similar calculations reveal the 
Table 4 FAS(2)

\begin{tabular}{c|ccccccccc|ccccccccc}
$\star$ & 0 & 1 & 2 & 3 & 4 & 5 & 6 & 7 & 8 & 9 & 10 & 11 & 12 & 13 & 14 & 15 & 16 & 17 \\
\hline 0 & 4 & 2 & 10 & 11 & 13 & 7 & 7 & 16 & 10 & 11 & 10 & 11 & 15 & 13 & 16 & 15 & 16 & 15 \\
1 & 3 & 5 & 8 & 9 & 12 & 6 & 6 & 14 & 8 & 9 & 8 & 9 & 17 & 12 & 14 & 17 & 14 & 17 \\
2 & 11 & 7 & 10 & 11 & 15 & 7 & 7 & 16 & 10 & 11 & 10 & 11 & 15 & 15 & 16 & 15 & 16 & 15 \\
3 & 12 & 8 & 8 & 9 & 12 & 14 & 14 & 14 & 8 & 9 & 8 & 9 & 17 & 12 & 14 & 17 & 14 & 17 \\
4 & 13 & 10 & 10 & 11 & 13 & 16 & 16 & 16 & 10 & 11 & 10 & 11 & 15 & 13 & 16 & 15 & 16 & 15 \\
5 & 9 & 6 & 8 & 9 & 17 & 6 & 6 & 14 & 8 & 9 & 8 & 9 & 17 & 17 & 14 & 17 & 14 & 17 \\
6 & 9 & 6 & 8 & 9 & 17 & 6 & 6 & 14 & 8 & 9 & 8 & 9 & 17 & 17 & 14 & 17 & 14 & 17 \\
7 & 11 & 7 & 10 & 11 & 15 & 7 & 7 & 16 & 10 & 11 & 10 & 11 & 15 & 15 & 16 & 15 & 16 & 15 \\
8 & 9 & 14 & 8 & 9 & 17 & 14 & 14 & 14 & 8 & 9 & 8 & 9 & 17 & 17 & 14 & 17 & 14 & 17 \\
\hline 9 & 17 & 8 & 8 & 9 & 17 & 14 & 14 & 14 & 8 & 9 & 8 & 9 & 17 & 17 & 14 & 17 & 14 & 17 \\
10 & 11 & 16 & 10 & 11 & 15 & 16 & 16 & 16 & 10 & 11 & 10 & 11 & 15 & 15 & 16 & 15 & 16 & 15 \\
11 & 15 & 10 & 10 & 11 & 15 & 16 & 16 & 16 & 10 & 11 & 10 & 11 & 15 & 15 & 16 & 15 & 16 & 15 \\
12 & 12 & 8 & 8 & 9 & 12 & 14 & 14 & 14 & 8 & 9 & 8 & 9 & 17 & 12 & 14 & 17 & 14 & 17 \\
13 & 13 & 10 & 10 & 11 & 13 & 16 & 16 & 16 & 10 & 11 & 10 & 11 & 15 & 13 & 16 & 15 & 16 & 15 \\
14 & 9 & 14 & 8 & 9 & 17 & 14 & 14 & 14 & 8 & 9 & 8 & 9 & 17 & 17 & 14 & 17 & 14 & 17 \\
15 & 15 & 10 & 10 & 11 & 15 & 16 & 16 & 16 & 10 & 11 & 10 & 11 & 15 & 15 & 16 & 15 & 16 & 15 \\
16 & 11 & 16 & 10 & 11 & 15 & 16 & 16 & 16 & 10 & 11 & 10 & 11 & 15 & 15 & 16 & 15 & 16 & 15 \\
17 & 17 & 8 & 8 & 9 & 17 & 14 & 14 & 14 & 8 & 9 & 8 & 9 & 17 & 17 & 14 & 17 & 14 & 17
\end{tabular}

size of $F A S(3)$ as 93, which motivates the following proposition and theorem. Much more computational data appears in [11].

Proposition 2.3 Words in normal form in F AS $(n)$ are characterized by the following family of words:

(1) Non-empty words in which every letter appears at most once,

(2) Words of the form aw, where $w$ is a word in which every letter appears at most once and the letter a appears exactly once, and

(3) Words obtained from words in (1) and (2) by doubling the last letter, in particular words of the form $a^{3}$, are possible.

Proof We refer to [11] for the proof. Here we only show how to reduce every word $w \in A_{n}^{\star} \backslash\{\emptyset\}$ to the normal form. Uniqueness can be proven by arguing along the lines of the proofs of Proposition 2.14 and Lemma 2.15.

Let $F A S(n)$ have generating set $A_{n}$ and let $w=b_{1} b_{2} \cdots b_{k} \in F A S(n)$ where $b_{i} \in A_{n}$ for all $1 \leq i \leq k$. If $w$ is already in one of the normal forms, we are done. Otherwise, we consider the remaining two possibilities. First, let $w$ be such that $b_{i}=b_{i+1}$ for some $1<i<k-1$. Then, by applying the reduction process illustrated in Eq. 2.1, we obtain a word with one fewer letter. Next, let $w$ be such that $b_{i}=b_{j}$ for some $1<i<j \leq k$ and $j-i>1$. Then, by applying right self-distributivity on $\left(b_{i-1} b_{i}\right)\left(\left(b_{i+1} \cdots b_{j-1}\right) b_{j}\right)$, we obtain a word with one fewer letter. Since $w$ has finite number of letters, we eventually reduce $w$ to one of the normal forms using a combination of the two reduction processes. 
Theorem 2.4 Let $|F A S(n)|$ be denoted by $c_{n}$. Then:

(1) $c_{n}$ is finite with cardinality $3 n+2 \sum_{i=2}^{n}(i+1) !\left(\begin{array}{c}n \\ i\end{array}\right)$

(2) $c_{n}$ satisfies the recursive relation $c_{n}=\frac{n^{2}}{n-1} c_{n-1}+n(n+1)$

(3) $c_{n}$ satisfies the 2-term recursive relation $c_{n}=(n+2) c_{n-1}-(n-1) c_{n-2}+3 n$

(4) $c_{n}$ has exponential generating function $\frac{\left(3 x-x^{3}\right) e^{x}}{(1-x)^{2}}$

Proof (1) This follows by a direct calculation from Proposition 2.3; we count words of length $i$ in the normal form (see also [11]).

(2) This formula can be derived from formulas given in [1], Appendix $2 .{ }^{6}$ We provide a proof for completeness.

$$
\text { Let } a_{n}=\sum_{i=0}^{n}(i+1) !\left(\begin{array}{l}
n \\
i
\end{array}\right) \text { and } b_{n}=\sum_{i=0}^{n}(i) !\left(\begin{array}{l}
n \\
i
\end{array}\right) \text {. }
$$

Then, $b_{n+1}-b_{n}=\sum_{i=1}^{n+1}(i) !\left(\begin{array}{c}n+1 \\ i\end{array}\right)-\sum_{i=1}^{n}(i) !\left(\begin{array}{l}n \\ i\end{array}\right)$

$$
\begin{aligned}
& =(n+1))+\sum_{i=1}^{n}(i) !\left(\left(\begin{array}{c}
n+1 \\
i
\end{array}\right)-\left(\begin{array}{l}
n \\
i
\end{array}\right)\right) \\
& =(n+1) !+\sum_{i=1}^{n}(i) !\left(\begin{array}{c}
n \\
i-1
\end{array}\right) \\
& =(n+1) !+\sum_{i=0}^{n-1}(i+1) !\left(\begin{array}{l}
n \\
i
\end{array}\right)=\sum_{i=0}^{n}(i+1) !\left(\begin{array}{l}
n \\
i
\end{array}\right)=a_{n} .
\end{aligned}
$$

Furthermore,

$$
\begin{aligned}
b_{n} & =1+\sum_{i=1}^{n}(i) !\left(\begin{array}{l}
n \\
i
\end{array}\right)=1+n \sum_{i=1}^{n}(n-1) \cdots(n-i+1) \\
& =1+n \sum_{i=0}^{n-1}(i) !\left(\begin{array}{c}
n-1 \\
i
\end{array}\right)=n b_{n-1}+1 .
\end{aligned}
$$

Thus, $a_{n}=b_{n+1}-b_{n}=n b_{n}+1=n^{2} b_{n-1}+n+1$ and $a_{n-1}=(n-1) b_{n-1}+1$. From these we conclude $(n-1) a_{n}=n^{2} a_{n-1}-1$. Since $c_{n}=2 a_{n}-n-2$, $(n-1) c_{n}=n^{2} c_{n-1}+(n-1) n(n+1)$.

(3) This follows from (2): $c_{n}=\frac{n^{2}}{n-1} c_{n-1}+n(n+1)=(n+2) c_{n-1}+\frac{2-n}{n-1} c_{n-1}+$ $n(n+1) \stackrel{(2)}{=}(n+2) c_{n-1}-(n-1) c_{n-2}+3 n$.

\footnotetext{
6 It is noted in that paper:"All statements given here are proved in [10]".
} 
Table 5 FPUS(2)

\begin{tabular}{l|cccccc}
$*$ & $a$ & $a^{2}$ & $a b$ & $b$ & $b^{2}$ & $b a$ \\
\hline$a$ & $a^{2}$ & $a^{2}$ & $a b$ & $a b$ & $a b$ & $b a$ \\
$a^{2}$ & $a^{2}$ & $a^{2}$ & $a b$ & $a b$ & $a b$ & $b a$ \\
$a b$ & $b a$ & $b a$ & $a b$ & $a b$ & $a b$ & $b a$ \\
$b$ & $b a$ & $b a$ & $a b$ & $b^{2}$ & $b^{2}$ & $b a$ \\
$b^{2}$ & $b a$ & $b a$ & $a b$ & $b^{2}$ & $b^{2}$ & $b a$ \\
$b a$ & $b a$ & $b a$ & $a b$ & $a b$ & $a b$ & $b a$
\end{tabular}

(4) This is straightforward from the theory of generating functions. We first observe that the generating function of the sequence $a_{n}$ is $\frac{e^{x}}{(1-x)^{2}}$.

\subsection{Free proto-unital shelves}

As noted before, any proto-unital shelf is associative but not vice versa.

The free proto-unital shelf F PUS(2) has six elements: $a, a^{2}, b, b^{2}, a b, b a$ and is depicted in Table 5.

For any alphabet $A$ we construct a proto-unital shelf $D(A)$ and show that it is the free proto-unital shelf over the alphabet $A$, using the observation that proto-unital shelves are associative.

Definition 2.5 Let $\widetilde{D}(A)$ be the set of nonempty words in $A^{*}$ which has no repeating letters and $D(A)=\widetilde{D}(A) \cup\left\{a^{2} \mid a \in A\right\}$. We define the binary operation $*$ on $D(A)$ as follows:

(1) If $w_{1}, w_{2} \in \widetilde{D}(A)$, and $w_{1} w_{2}$ (juxtaposition) has at least two different letters then $w_{1} * w_{2}$ is obtained from $w_{1} w_{2}$ by deleting from $w_{1}$ all letters which are also in $w_{2}$.

(2) Otherwise we have: $a^{2} * a^{2}=a^{2}$, and $w_{1} * a^{2}=w_{1} * a, a^{2} * w_{2}=a * w_{2}$ for $a \in A$ and $w_{i} \in \widetilde{D}(A)$. In particular, $a^{2} * a=a^{2}=a * a^{2}$.

Proposition 2.6 $D(A)$ is a proto-unital shelf isomorphic to the free proto-unital shelf over the alphabet A, FPUS(A).

Before proving the above proposition we need the following three lemmas.

Lemma 2.7 Let $f_{A}: A^{\star} \backslash\{\emptyset\} \rightarrow D(A)$ be a map given by:

(i) $f\left(a^{k}\right)=a^{2}$ for $k \geq 2, a \in A^{\star} \backslash\{\emptyset\}$.

(ii) Otherwise if $w \neq a^{k}, k \geq 2, f(w)$ is the word obtained from $w \in A^{\star} \backslash\{\varnothing\}$ by deleting repeating letters, leaving only the last occurrence. Then,

(1) $f_{A}$ is a retraction, that is $f_{A} \circ e=I d_{D(A)}$ where $e$ is the natural embedding of $D(A)$ in to $A^{\star} \backslash\{\emptyset\}$.

(2) $f_{A}\left(w_{1} w_{2}\right)=f_{A}\left(w_{1}\right) * f_{A}\left(w_{2}\right)$, that is $f_{A}$ is a homomorphism. 
(3) $D(A)$ is associative.

Proof Parts (1) and (2) follow immediately from Definition 2.5. Part (3) follows from parts (1) and (2) and the fact that $A^{*} \backslash\{\emptyset\}$ is associative.

Lemma 2.8 (1) $f_{A}$ is a shelf epimorphism, and

(2) $f_{A}$ descends to a shelf isomorphism $\hat{f}_{A}: F P U S(A) \longrightarrow D(A)$.

Proof (1) First we check that $D(A)$ is self-distributive. Namely, if $w_{1}, w_{2}, w_{3} \in \widetilde{D}(A)$ and $w_{1} w_{2} w_{3}$ uses at least two different letters, then $\left(w_{1} * w_{2}\right) * w_{3}$ is obtained from $w_{1} w_{2} w_{3}$ by deleting from $w_{1}$ letters which are already in $w_{2}$ and deleting from $w_{1} * w_{2}$ letters which are already in $w_{3}$. When using the rules for $*$ operation we see that $\left(w_{1} * w_{2}\right) * w_{3}$ and $\left(w_{1} * w_{3}\right) *\left(w_{2} * w_{3}\right)$ give the same result. If $w_{1} w_{2} w_{3}$ uses only one letter, say $a$ then $\left(w_{1} * w_{2}\right) * w_{3}=a^{2}=\left(w_{1} * w_{3}\right) *\left(w_{2} * w_{3}\right)$. We are left to consider the case where one of $w_{i}, i=1,2,3$ is equal to $a^{2}$ for $a \in A$. By the definition of the multiplication, $w_{i}=a^{2}$ can be replaced by $w_{i}=a$ without changing neither $\left(w_{1} * w_{2}\right) * w_{3}$ nor $\left(w_{1} * w_{3}\right) *\left(w_{2} * w_{3}\right)$. General self-distributivity follows from the case where $w_{1}, w_{2}, w_{3} \in \widetilde{D}(A)$.

(2) Since $f_{A}\left(a b^{2}\right)=f_{A}(a b)$ and $f_{A}(a w a)=f_{A}(w a), f_{A}$ descends to the shelf isomorphism $\hat{f}_{A}: F \tilde{P} U S(A) \longrightarrow \widetilde{D}(A)$.

Lemma 2.9 (1) Every element of $A^{\star} \backslash\{\emptyset\} / \sim_{0}$, where $\sim_{0}$ is generated by relations $a b^{2}=a b$ and $a b a=b a, a, b \in A$, has the unique representative in $D(A)$ called the normal form of the word $w$.

(2) $A^{\star} \backslash\{\emptyset\} / \sim=A^{\star} \backslash\{\emptyset\} / \sim_{0}$. In other words, self-distributivity and axioms $a * b=$ $b *(a * b)$ and $a * b=(a * b) * b$ follow from $a b^{2}=a b$ and $a b a=b a$.

Proof Part (2) First we show, by induction on the length of $w$, that $a w a=w a$ for $a \in A$ and $w \in A^{*}-\emptyset$. The proof is straightforward and the inductive step is as follows: for $w=w_{0} b$ we have $w a=\left(w_{0} b\right) a=\left(w_{0} a\right) b a=a w_{0}(a b a)=a\left(w_{0} b\right) a=a w a$. Now let $v, w \in A^{\star} \backslash\{\emptyset\}$. Then $v * w=w *(v * w)$ and $v * w=(v * w) * w$ follow by induction on the length of the words and identities $a b^{2}=a b$ and $a w a=w a$. Self-distributivity follows from part 2 of Remark 1.4.

For part (1) the main ingredient used here is the reduction of the word $a^{2} b$, because the number of letters actually increases before reducing:

$$
a^{2} b=b\left(a^{2} b\right)=((b a) a) b=b(a b)=a b
$$

Using this and axioms $a b^{2}=a b$ and $a w a=w a$, we can shorten every word which is not in $D(A)$ and in finite number of steps find a representative in $D(A)$.

We continue with the proof of Proposition 2.6.

Proof The homomorphism $f_{A}$ descends to $\hat{f}_{A}: A^{\star} \backslash\{\emptyset\} / \sim_{0} \longrightarrow D(A)$ because $f_{A}\left(a b^{2}\right)=f_{A}(a b)$ and $f_{A}(a w a)=f_{A}(w a)$. By Lemma $2.9 \hat{f}_{A}$ is an isomorphism between $F P U S(A)$ and $D(A)$. It follows that $D(A)$ is also a proto-unital shelf. 
Table $6 F \widetilde{P} U S(2)$

\begin{tabular}{c|cccc}
$*$ & $a$ & $b$ & $a b$ & $b a$ \\
\hline$a$ & $a$ & $a b$ & $a b$ & $b a$ \\
$b$ & $b a$ & $b$ & $a b$ & $b a$ \\
$a b$ & $b a$ & $a b$ & $a b$ & $b a$ \\
$b a$ & $b a$ & $a b$ & $a b$ & $b a$
\end{tabular}

Theorem 2.10 Let F PUS $(A)$ be the free proto-unital shelf over the alphabet A. Then:

(1) If $A=A_{n}$ is a finite alphabet of $n$ letters then,

$$
|F P U S(n)|=n+\sum_{k=1}^{n} k !\left(\begin{array}{l}
n \\
k
\end{array}\right)=n-1+\sum_{k=0}^{n} k !\left(\begin{array}{l}
n \\
k
\end{array}\right)
$$

(2) The exponential generating function of $|F P U S(n)|$ is $\left(2 x-x^{2}\right) \frac{e^{x}}{1-x}$

Proof (1) This follows from counting elements in normal form.

(2) This follows from a straightforward generating functions computation.

Definition 2.11 Let $(X, *)$ be a magma. If there exists elements $c, r \in X$ such that $x * r=c$ for all $x \in X$ then $r$ is a right $c$-fixed element. If $r=c$ then $r(=c)$ is a right zero or right projector.

Corollary 2.12 FPUS(n), has $n$ ! different right zeros (right projectors), namely:

(1) $a^{2}$ for $n=1$, and

(2) $a_{i_{1}} a_{i_{2}} \cdots a_{i_{n}}$ where $a_{i_{1}}, a_{i_{2}}, \ldots, a_{i_{n}}$ is a permutation of alphabet letters from $A_{n}$.

Proof This follows from the structure of multiplication of words in normal form as described in the proof of Lemma 2.9.

\subsection{Free pre-unital and unital shelves}

Free pre-unital shelves and unital shelves are discussed together because, by the result of Proposition 1.7, they differ only by one element (namely the empty word in the presentation of the shelf by generators and relators).

The free pre-unital shelf $F \widetilde{P} U S(2)$ is illustrated in Table 6.

For any alphabet $A$ we construct a pre-unital shelf $\widetilde{D}(A)$ and show that it is the free pre-unital shelf over the alphabet $A$, using the observation that pre-unital shelves are associative.

Definition 2.13 Let $A$ be a set and $\widetilde{D}(A)$ be the set of non-empty words in $A^{*}$ which have no repeating letters. We define the binary operation $*$ on $\widetilde{D}(A)$ by: If $w_{1}, w_{2} \in$ $\widetilde{D}(A)$ then $w_{1} * w_{2}$ is obtained from $w_{1} w_{2}$ (juxtaposition) by deleting from $w_{1}$ all letters which are also in $w_{2}$. 
Proposition 2.14 $\widetilde{D}(A)$ is a pre-unital shelf isomorphic to the free pre-unital shelf over the alphabet $A, F \widetilde{P} U S(A)$.

Proof First we show that $\widetilde{D}(A)$ is a pre-unital shelf. Associativity is verified directly from definition. Namely, $\left(w_{1} * w_{2}\right) * w_{3}$ is obtained from $w_{1} w_{2} w_{3}$ by deleting from $w_{1}$ letters which are already in $w_{2}$ and deleting from $w_{1} * w_{2}$ letters which are already in $w_{3}$.

Furthermore, from the rule $w_{1} * w_{1}=w_{1}$, idempotence follows immediately as does axiom (1) in Proposition 1.3: $w_{2} *\left(w_{1} * w_{2}\right)=w_{1} * w_{2}$. Axiom (2) in Proposition 1.3 follows from associativity and idempotence: $\left(w_{1} * w_{2}\right) * w_{2}=w_{1} *\left(w_{2} * w_{2}\right)=w_{1} * w_{2}$. Self-distributivity follows from Remark 1.4.

The second part of the proposition follows by the following lemma which describes the concrete isomorphism from $F \tilde{P} U S(A)$ onto $\widetilde{D}(A)$.

Lemma 2.15 Let $f_{A}: A^{*} \backslash\{\emptyset\} \rightarrow \widetilde{D}(A)$ be a map (retraction) given by: $f(w)$ is the word obtained from $w \in A^{*} \backslash\{\emptyset\}$ by deleting repeating letters, leaving only the last occurrence; $\widetilde{D}(A)$ embeds in $A^{*} \backslash\{\emptyset\}$ and $f$ is constant on the image of $\widetilde{D}(A)$. Then:

(1) $f_{A}$ is a shelf epimorphism, and

(2) $f_{A}$ descends to an isomorphism $\hat{f}_{A}: F \tilde{P} U S(A) \longrightarrow \widetilde{D}(A)$.

For any $w \in A_{n}^{\star} \backslash\{\emptyset\}, f(w) \in \widetilde{D}(A)$ is called the normal form of $w$.

Proof (1) The equality $f_{A}\left(w_{1} w_{2}\right)=f_{A}\left(w_{1}\right) * f_{A}\left(w_{2}\right)$ follows from the definition of the function $f_{A}$. That is, only the last occurrence of a given letter survives in $f_{A}\left(w_{1} w_{2}\right)$ and $f_{A}\left(w_{1}\right) * f_{A}\left(w_{2}\right)$. Thus, $f_{A}$ is a shelf homomorphism.

(2) Since $f_{A}(b b)=f_{A}(b)$ and $f_{A}(b w b)=f_{A}(w b), f_{A}$ descends to the map $\hat{f}_{A}$ : $F \tilde{P} U S(A) \longrightarrow \tilde{D}(A)$.

To conclude that $f_{A}$ is an isomorphism we notice that any $w \in A^{*} \backslash\{\emptyset\}$ can be reduced to an element without letter repetition by relations in $\sim$. That is, $u b w b v=$ $u w b v$, for any $u, w, v \in A^{*}$ (used for any repeated letter, $b$ ).

From Proposition 2.14 we can calculate the number of elements in $F \widetilde{P} U S(n)$ and its exponential generating function.

Corollary 2.16 Let $|F \widetilde{P} U S(n)|$ be denoted by $\bar{b}_{n}$. Then:

(1) $\bar{b}_{n}=\sum_{i=1}^{n} n(n-1) \cdots(n-i+1)=\sum_{i=1}^{n} i !\left(\begin{array}{c}n \\ i\end{array}\right)$

(2) $\bar{b}_{n}$ satisfies the recursion relation $\bar{b}_{n}=n \bar{b}_{n-1}+n$ with $\bar{b}_{0}=0, \bar{b}_{1}=1, \bar{b}_{2}=$ $4, \bar{b}_{3}=15, \bar{b}_{4}=64, \bar{b}_{5}=325, \bar{b}_{6}=1956$.

(3) $\bar{b}_{n}$ has exponential generating function $\sum_{i=0}^{\infty} \bar{b}_{i} \frac{x^{i}}{i !}=\left(\frac{e^{x}}{1-x}-e^{x}\right)=e^{x}\left(\frac{x}{1-x}\right)$

Proof (1) The irreducible (normal form) words in $F \widetilde{P} U S(n)$ are all arrangements of $i$ different letters of the alphabet of $n$ letters (empty word excluded). We calculate the number as: $n+n(n-1)+\cdots+n(n-1) \cdots(n-i+1)+\cdots+n !=\sum_{i=1}^{n} i !\left(\begin{array}{c}n \\ i\end{array}\right)$, as desired.

(2) This follows directly from the formula in (1). Notice that $\bar{b}_{n}=b_{n}-1$ where $b_{n}$ was considered in the proof of Theorem 2.4. Thus the recurrence relation (2) follows from $b_{n}=n b_{n-1}+1$. 
(3) This follows from a straightforward generating functions computation: we observe first that the generating function of the sequence $b_{n}=\sum_{i=0}^{n} i !\left(\begin{array}{c}n \\ i\end{array}\right)$ is $\frac{e^{x}}{(1-x)}$.

The free unital shelf $F U S(n)$ is obtained from $F \widetilde{P} U S(n)$ using Proposition 1.7 by adding empty word. Therefore, we have the following:

Proposition 2.17 (1) The number of elements in FUS $(n)$ is one more than the number of elements in F $\widetilde{P} U S(n)$. That is, $|F U S(n)|=\sum_{i=0}^{n} i !\left(\begin{array}{c}n \\ i\end{array}\right)$

(2) The exponential generating function for $|F U S(n)|$ is $\frac{e^{x}}{1-x}$

Remark 2.18 The material of this subsection was essentially proven by Kimura in 1958 [13] where he considered monoids satisfying the axiom $a * b=b * a * b$ (where $a$ can be the empty word). It was proven that free monoids with this axiom consist of words without repeating letters, hence in the case of $n$ generators, he obtained $\sum_{i=0}^{n} i !\left(\begin{array}{c}n \\ i\end{array}\right)$ elements.

\section{Homology of associative shelves}

In this section, we first recall the definition of one-term distributive homology introduced in [22] and two-term homology introduced in [7-9]. Following [18], we first define the chain modules (denoted by $C_{n}$ for $n \geq 0$ ) and the face maps $d_{i, n}: C_{n} \longrightarrow C_{n-1}$, satisfying $d_{i, n-1} \circ d_{j, n}=d_{j-1, n-1} \circ d_{i, n}$ for $0 \leq i \leq n, n \geq 1$, and $i<j$, so that $\left(C_{n}, d_{i, n}\right)$ forms a pre-simplicial module. After defining the boundary map $\partial_{n}: C_{n} \longrightarrow C_{n-1}$ by $\partial_{n}=\sum_{i=0}^{n}(-1)^{i} d_{i, n}$, we obtain the chain complex $\mathcal{C}=\left(C_{n}, \partial_{n}\right)$.

We describe two pre-simplicial modules leading up to one-term and two-term homology, respectively. Let $(X, *)$ be a shelf and $C_{n}$ the free abelian group generated by the $(n+1)$-tuples $\left(x_{0}, x_{1}, \ldots, x_{n}\right)$ of the elements in $X$, that is $C_{n}=\mathbb{Z} X^{n+1}$. By convention, $C_{-1}=\{0\}$ and $d_{0,0}=0$.

We begin with the pre-simplicial module leading to one-term homology. For $0 \leq$ $i \leq n$, let $d_{i, n}^{(*)}: C_{n} \longrightarrow C_{n-1}$ be given on the basis $X^{n+1}$ by:

$d_{i, n}^{(*)}\left(\left(x_{0}, x_{1}, \cdots, x_{n}\right)\right)= \begin{cases}\left(x_{1}, x_{2}, \ldots, x_{n}\right) & \text { if } \mathrm{i}=0, \\ \left(x_{0} * x_{i}, x_{1} * x_{i}, \ldots, x_{i-1} * x_{i}, x_{i+1}, \ldots, x_{n}\right) & 0<\mathrm{i}<\mathrm{n}, \\ \left(x_{0} * x_{n}, x_{1} * x_{n}, \ldots, x_{n-1} * x_{n}\right) & \text { if } \mathrm{i}=\mathrm{n} .\end{cases}$

The map $d_{i, n}^{(*)}$ is extended linearly from $X^{n+1}$ to $C_{n}$.

We then have $d_{i, n-1}^{(*)} \circ d_{j, n}^{(*)}=d_{j-1, n-1}^{(*)} \circ d_{i, n}^{(*)}$, and therefore $\left(C_{n}, d_{i, n}^{(*)}\right)$ is a presimplicial module. Defining the boundary map as usual by $\partial_{n}^{(*)}=\sum_{i=0}^{n}(-1)^{i} d_{i, n}^{(*)}$, $\mathcal{C}^{(*)}=\left(C_{n}, \partial_{n}^{(*)}\right)$ is a chain complex whose $n^{\text {th }}$ homology group is given by:

$$
H_{n}^{(*)}(X)=\frac{\operatorname{ker}\left(\partial_{n}^{(*)}\right)}{i m\left(\partial_{n+1}^{(*)}\right)} .
$$


To define two-term homology, we consider the trivial operation given by $a *_{0}$ $b=a$. Notice that $d_{i, n}^{\left(*_{0}\right)}\left(\left(x_{0}, x_{1}, \ldots, x_{n}\right)\right)=\left(x_{0}, x_{1}, \ldots, x_{i-1}, x_{i+1}, x_{i+2}, \ldots, x_{n}\right)$. We define face maps $d_{i, n}^{R}: C_{n} \longrightarrow C_{n-1}$ by $d_{i, n}^{R}=d_{i, n}^{\left(*_{0}\right)}-d_{i, n}^{(*)}$. We then have $d_{i, n-1}^{R} \circ d_{j, n}^{R}=d_{j-1, n-1}^{R} \circ d_{i, n}^{R}$, hence $\left(C_{n}, d_{i, n}^{R}\right)$ is a pre-simplicial module. Defining the boundary map as usual by $\partial_{n}^{R}=\sum_{i=0}^{n}(-1)^{i} d_{i, n}^{R}, \mathcal{C}^{R}=\left(C_{n}, \partial_{n}^{R}\right)$ is a chain complex whose $n^{\text {th }}$ two-term homology group is given by:

$$
H_{n}^{R}(X)=\frac{\operatorname{ker}\left(\partial_{n}^{R}\right)}{i m\left(\partial_{n+1}^{R}\right)} .
$$

We now introduce the main results of the paper on one-term and two-term homology of unital shelves. The following theorem in the context of one-term homology appears in $[22,24]$.

Theorem 3.1 ([22]) If one of the following conditions holds:

(1) $x \longmapsto x * y$ is a bijection on $X$ for some $y$, or

(2) there is $y \in X$ such that $y * x=y$ for all $x \in X$,

then $\widetilde{H}_{n}^{(*)}(X)=0$ for all $n .^{7}$

There are many associative shelves satisfying condition (2) of Theorem 3.1. All of them have trivial one-term homology in all dimensions. Furthermore, applying Theorem 3.1 gives:

Theorem 3.2 Unital shelves have trivial one-term homology in all positive dimensions. That is $\widetilde{H}^{(*)}(X)=0$, for all $n \geq 0$.

Proof The existence of the unit element in unital shelves implies condition (1) of Theorem 3.1 is satisfied.

Before stating our main result for two-term homology, namely Theorem 3.3, we remark that if $r$ is a right $c$-fixed element in a shelf $(X, *)$, then $c=c * r=(c * r) * r=$ $(c * r) *(r * r)=c * c$. Therefore, $c$ is idempotent.

Theorem 3.3 Two-term homology of shelves with a right fixed element is $\mathbb{Z}$ in all dimensions.

The proof of the above theorem follows from the following three lemmas.

\footnotetext{
7 We note that $\widetilde{H}_{n}^{(*)}$ denotes the augmented one-term homology obtained from $\mathcal{C}^{(*)}=\left(C_{n}, \partial_{n}^{(*)}\right)_{n \geq 0}$ by defining $C_{-1}=\mathbb{Z}$ and $\partial_{0}^{(*)}(x)=1$ on the basis $X$. The composite $\partial_{0}^{(*)} \circ \partial_{1}^{(*)}=0$, so that the augmented distributive chain complex $\widetilde{\mathcal{C}}^{(*)}$ and augmented one-term distributive homology $\widetilde{H}_{n}^{(*)}$ satisfies:

$$
H_{n}^{(*)}(X)= \begin{cases}\mathbb{Z} \oplus \widetilde{H}_{n}^{(*)}(X) & \text { if } n=0 \\ \widetilde{H}_{n}^{(*)}(X) & \text { if } n \geq 1\end{cases}
$$
}


Lemma 3.4 Let $(X, *)$ be a shelf with a right c-fixed element $r$. Let $\mathcal{C}^{R}=\left(C_{n}, \partial_{n}^{R}\right)$ be the chain complex corresponding to the two-term homology of $X$. Then, there exists a sequence of maps $f_{n}: \mathbb{Z} X^{n} \longrightarrow \mathbb{Z} X^{n+1}$ which is a chain homotopy between the identity on $C_{n}$ and the constant map on $C_{n}, \alpha_{n}^{c}: C_{n} \longrightarrow C_{n}$, given by $\alpha_{n}^{c}\left(x_{0}, x_{1}, \ldots, x_{n}\right)=$ $(c, c, \ldots, c)$.

Proof Let $(X, *)$ be a shelf with a right $c$-fixed element $r$. Let $\mathcal{C}^{R}=\left(C_{n}, \partial_{n}^{R}\right)$ be the chain complex corresponding to the two-term homology of $X$. Consider a sequence of maps (chain homotopy) $f_{n}: \mathbb{Z} X^{n} \longrightarrow \mathbb{Z} X^{n+1}$, for all positive integers $n$, given by:

$$
f\left(x_{0}, x_{1}, \ldots, x_{n}\right)=(-1)^{n+1}\left(x_{0}, x_{1}, \ldots, x_{n}, r\right) .
$$

We will show that this sequence of maps is a chain homotopy between the identity chain map and the constant chain map $\left(x_{0}, x_{1}, \ldots, x_{n}\right) \longmapsto(c, c, \ldots, c)$.

Consider the complex:

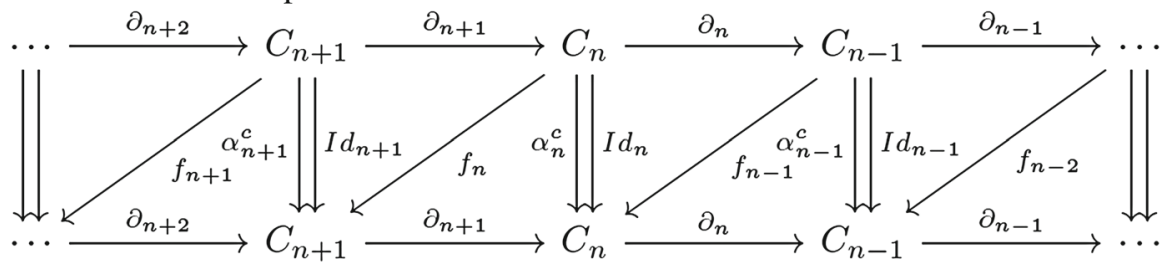

Then:

$$
\begin{aligned}
& \left(\partial_{n+1}^{R} \circ f_{n}\right)\left(x_{0}, x_{1}, \ldots, x_{n}\right)=(-1)^{n+1} \partial_{n+1}^{R}\left(x_{0}, x_{1}, \ldots, x_{n}, r\right) \\
& =(-1)^{n+1}\left\{\sum_{i=1}^{n}(-1)^{i}\left(x_{0}, x_{1}, \ldots, x_{i-1}, x_{i+1}, x_{i+2}, \ldots, x_{n}, r\right)\right\}+\left(x_{0}, x_{1}, \ldots, x_{n}\right) \\
& \quad-(-1)^{n+1}\left\{\sum_{i=1}^{n}(-1)^{i}\left(x_{0} * x_{i}, x_{1} * x_{i}, \ldots, x_{i-1} * x_{i}, x_{i+1}, x_{i+2}, \ldots, x_{n}, r\right)\right\} \\
& \quad-(c, c, \ldots, c),
\end{aligned}
$$

and

$$
\begin{aligned}
& \left(f_{n-1} \circ \partial_{n}^{R}\right)\left(x_{0}, x_{1}, \ldots, x_{n}\right)=f_{n-1}\left(\sum_{i=1}^{n}(-1)^{i}\left(x_{0}, x_{1}, \ldots, x_{i-1}, x_{i+1}, x_{i+2}, \ldots, x_{n}\right)\right. \\
& \left.\quad-\sum_{i=1}^{n}(-1)^{i}\left(x_{0} * x_{i}, x_{1} * x_{i}, \ldots, x_{i-1} * x_{i}, x_{i+1}, x_{i+2}, \ldots, x_{n}\right)\right) \\
& =(-1)^{n}\left\{\sum_{i=1}^{n}(-1)^{i}\left(x_{0}, x_{1}, \ldots, x_{i-1}, x_{i+1}, x_{i+2}, \ldots, x_{n}, r\right)\right\} \\
& \quad-(-1)^{n}\left\{\sum_{i=1}^{n}(-1)^{i}\left(x_{0} * x_{i}, x_{1} * x_{i}, \ldots, x_{i-1} * x_{i}, x_{i+1}, x_{i+2}, \ldots, x_{n}, r\right)\right\}
\end{aligned}
$$

Taking the sum of the two equations above we get

$$
\begin{aligned}
& \left(\partial_{n+1}^{R} \circ f_{n}\right)\left(x_{0}, x_{1}, \ldots, x_{n}\right)+\left(f_{n-1} \circ \partial_{n}^{R}\right)\left(x_{0}, x_{1}, \ldots, x_{n}\right) \\
& \quad=\left(x_{0}, x_{1}, \ldots, x_{n}\right)-(c, c, \ldots, c)
\end{aligned}
$$

that is, $\partial^{R} \circ f+f \circ \partial^{R}=I d-\alpha^{c}$. 
Table 7 An associative shelf which is not a proto-unital shelf but has right zeros

\begin{tabular}{l|llll}
$*$ & 0 & 1 & 2 & 3 \\
\hline 0 & 0 & 0 & 2 & 3 \\
1 & 0 & 0 & 2 & 3 \\
2 & 0 & 0 & 2 & 3 \\
3 & 0 & 2 & 2 & 3
\end{tabular}

Lemma 3.5 Let $(X, *)$ be a shelf with a right $c$-fixed element $r$ for some $c \in X$. Let $\mathcal{C}^{R^{c}}$ be the sub-chain complex of $\mathcal{C}^{R}$, where $C_{n}^{c}$ is spanned by $(c, c, \ldots, c)$. Then, $H_{n}^{R}\left(\mathcal{C}^{R^{c}}\right)=\mathbb{Z}$.

Proof We have $C_{n}^{c}=\mathbb{Z}$ and the boundary maps are zero maps. Therefore, $H_{n}^{R}\left(\mathcal{C}^{R^{c}}\right)=$ $C_{n}^{c}=\mathbb{Z}$.

Lemma 3.6 $\mathcal{C}^{R}$ is chain homotopy equivalent to $\mathcal{C}^{R^{c}}$. In particular, they have the same homology groups.

Proof Let $\gamma_{n}^{c}: C_{n} \longrightarrow C_{n}^{c}$, be given by $\gamma_{n}^{c}\left(x_{0}, x_{1}, \ldots, x_{n}\right)=(c, c, \ldots, c)$ and let $\beta_{n}^{c}: C_{n}^{c} \longrightarrow C_{n}$ be an embedding given by $\beta_{n}^{c}(c, c, \ldots, c)=(c, c, \ldots, c)$. Then, $\gamma_{n}^{c} \circ \beta_{n}^{c}=I d_{C_{n}^{c}}$ and by Lemma 3.4, $\beta_{n}^{c} \circ \gamma_{n}^{c}$ is homotopy equivalent to $I d_{C_{n}}$. Therefore, these chain complexes are chain homotopy equivalent.

The universal property of free proto-unital shelves FPUS $(n)$ together with Corollary 2.12 gives the following proposition.

Proposition 3.7 Finitely generated proto-unital shelves have right zero elements.

Proof Let $(X, *)$ be a finitely generated proto-unital shelf with $n$ generators. Then there exists an epimorphism from $F P U S(n)$ onto $X$. Therefore, the images of right zero elements in F PUS $(n)$ are right zeros in $X$.

Note that the converse of the above proposition is not true. Table 7 gives an example of an associative shelf that is not a proto-unital shelf, as we have $(3 * 1) * 1=2 * 1=$ $0 \neq 2=3 * 1$.

This brings us to a stronger analog of Theorem 3.2 for two-term homology:

Theorem 3.8 Two-term homology of finitely generated proto-unital shelves is $\mathbb{Z}$ in all dimensions.

For the free algebraic structures discussed in the previous section, we have the corollary:

Corollary 3.9 (1) FUS(n) has trivial one-term homology in all positive dimensions for all $n$.

(2) FPUS(n) has two-term homology $\mathbb{Z}$ in all dimensions for any $n$. Hence, in particular, the same holds for $F \widetilde{P} U S(n)$ and FUS(n) as well.

We continue with two applications of Theorem 3.3. Recall that a spindle is a magma satisfying the idempotence and self-distributivity properties of a quandle. 
Definition 3.10 ([3]) Choose a family of sets $\left\{X_{i}\right\}_{i \in I}$, not necessarily finite, and functions $f_{i}: X_{i} \longrightarrow X_{i}$. Define the spindle product on $X=\bigsqcup_{i \in I} X_{i}$ for $x \in X_{i}$ and $y \in X_{j}$ by:

$$
x * y= \begin{cases}y, & \text { if } i=j \\ f_{j}(y), & \text { otherwise }\end{cases}
$$

Subsets $X_{i} \subset X$ are called blocks of the spindle $X$ and the maps $f_{i}$ are called block functions. We write $f: X \longrightarrow X$ for the function induced by all the block functions.

One-term homology was initially thought to be torsion-free and hence less interesting than two-term homology. In [24], there are several examples in which families of shelves are constructed. In [3], the one-term homology of one such infinite family was studied. Theorem 5.4 in [3] states that any finite abelian group can be realized as the torsion subgroup of the second homology group of $f$-block spindles having a one element block. But, every $f$-block spindle with a one element block has a right zero element.

Therefore, there exist infinite families of spindles which can realize any finite commutative group as a torsion subgroup in one-term homology. But, they have $\mathbb{Z}$ as their two-term homology group in all dimensions.

Theorems 3.1 and 3.3 can be further applied to determine the one-term and two-term homology groups of Laver tables. Richard Laver discovered these algebraic structures while studying set theory, and the combinatorial structure of Laver tables has been extensively studied $[4,5,16]$.

In the following discussion, we use the convention of Laver $[4,5,14,16]$, that is, the magmas are left self-distributive. To study Laver tables as right self-distributive algebraic structures the corresponding operation tables have to be transposed.

Definition 3.11 A Laver table, denoted by $A_{n}$, is the unique shelf $\left(\left\{1,2, \cdots, 2^{n}\right\}, *\right)$ for any positive integer $n$ satisfying $a * 1=1+a\left(\bmod 2^{n}\right)$.

The uniqueness in this definition was proven by Richard Laver in 1992. Tables 8, 9 , and 10 provide examples and satisfy left self-distributivity.

The element $2^{n}-1$ in the Laver table $A_{n}$ (after transposing) is a right fixed element. Further, the element $2^{n}$ is an element with respect to which $A_{n}$ (after transposing) satisfies condition (1) of Theorem $3.1[4,16]$. Therefore, the following result holds:

Table 8 The Laver table $A_{2}$

\begin{tabular}{c|cccc}
$*$ & 1 & 2 & 3 & 4 \\
\hline 1 & 2 & 4 & 2 & 4 \\
2 & 3 & 4 & 3 & 4 \\
3 & 4 & 4 & 4 & 4 \\
4 & 1 & 2 & 3 & 4
\end{tabular}


Table 9 The Laver table $A_{3}$

\begin{tabular}{c|cccccccc}
$*$ & 1 & 2 & 3 & 4 & 5 & 6 & 7 & 8 \\
\hline 1 & 2 & 4 & 6 & 8 & 2 & 4 & 6 & 8 \\
2 & 3 & 4 & 7 & 8 & 3 & 4 & 7 & 8 \\
3 & 4 & 8 & 4 & 8 & 4 & 8 & 4 & 8 \\
4 & 5 & 6 & 7 & 8 & 5 & 6 & 7 & 8 \\
5 & 6 & 8 & 6 & 8 & 6 & 8 & 6 & 8 \\
6 & 7 & 8 & 7 & 8 & 7 & 8 & 7 & 8 \\
7 & 8 & 8 & 8 & 8 & 8 & 8 & 8 & 8 \\
8 & 1 & 2 & 3 & 4 & 5 & 6 & 7 & 8
\end{tabular}

Table 10 The Laver table $A_{4}$

\begin{tabular}{c|cccccccc|cccccccc}
$*$ & 1 & 2 & 3 & 4 & 5 & 6 & 7 & 8 & 9 & 10 & 11 & 12 & 13 & 14 & 15 & 16 \\
\hline 1 & 2 & 12 & 14 & 16 & 2 & 12 & 14 & 16 & 2 & 12 & 14 & 16 & 2 & 12 & 14 & 16 \\
2 & 3 & 12 & 15 & 16 & 3 & 12 & 15 & 16 & 3 & 12 & 15 & 16 & 3 & 12 & 15 & 16 \\
3 & 4 & 8 & 12 & 16 & 4 & 8 & 12 & 16 & 4 & 8 & 12 & 16 & 4 & 8 & 12 & 16 \\
4 & 5 & 6 & 7 & 8 & 13 & 14 & 15 & 16 & 5 & 6 & 7 & 8 & 13 & 14 & 15 & 16 \\
5 & 6 & 8 & 14 & 16 & 6 & 8 & 14 & 16 & 6 & 8 & 14 & 16 & 6 & 8 & 14 & 16 \\
6 & 7 & 8 & 15 & 16 & 7 & 8 & 15 & 16 & 7 & 8 & 15 & 16 & 7 & 8 & 15 & 16 \\
7 & 8 & 16 & 8 & 16 & 8 & 16 & 8 & 16 & 8 & 16 & 8 & 16 & 8 & 16 & 8 & 16 \\
8 & 9 & 10 & 11 & 12 & 13 & 14 & 15 & 16 & 9 & 10 & 11 & 12 & 13 & 14 & 15 & 16 \\
\hline 9 & 10 & 12 & 14 & 16 & 10 & 12 & 14 & 16 & 10 & 12 & 14 & 16 & 10 & 12 & 14 & 16 \\
10 & 11 & 12 & 15 & 16 & 11 & 12 & 15 & 16 & 11 & 12 & 15 & 16 & 11 & 12 & 15 & 16 \\
11 & 12 & 16 & 12 & 16 & 12 & 16 & 12 & 16 & 12 & 16 & 12 & 16 & 12 & 16 & 12 & 16 \\
12 & 13 & 14 & 15 & 16 & 13 & 14 & 15 & 16 & 13 & 14 & 15 & 16 & 13 & 14 & 15 & 16 \\
13 & 14 & 16 & 14 & 16 & 14 & 16 & 14 & 16 & 14 & 16 & 14 & 16 & 14 & 16 & 14 & 16 \\
14 & 15 & 16 & 15 & 16 & 15 & 16 & 15 & 16 & 15 & 16 & 15 & 16 & 15 & 16 & 15 & 16 \\
15 & 16 & 16 & 16 & 16 & 16 & 16 & 16 & 16 & 16 & 16 & 16 & 16 & 16 & 16 & 16 & 16 \\
16 & 1 & 2 & 3 & 4 & 5 & 6 & 7 & 8 & 9 & 10 & 11 & 12 & 13 & 14 & 15 & 16
\end{tabular}

Corollary 3.12 Let $A_{n}^{t}$ denote the Laver table $A_{n}$ after converting to right selfdistributive magmas (by transposing). Then

(1) $\widetilde{H}_{n}^{(*)}\left(A_{n}^{t}\right)=0$, for all $n \geq 0$, and

(2) $H_{n}^{R}\left(A_{n}^{t}\right)=\mathbb{Z}$, for all $n \geq 0$ ([16], Theorem $\left.A\right)$.

\section{Future directions}

The results contained here regarding one-term and two-term homology for special families of associative shelves naturally lead to a desire to better understand the role of associativity in self-distributive algebraic structures. These preliminary computations lead to the conjectures that follow. Additionally some computational data appears in the Appendix. 
Conjecture 4.1 Associative shelves have no torsion in one-term homology.

Conjecture 4.2 Associative shelves have no torsion in two-term homology.

Acknowledgements The authors would like to thank Maciej Niebrzydowski for initial computations and Marithania Silvero for useful comments and suggestions. Furthermore, the authors would like to thank the referee for comments and suggestions.

\section{Appendix: computer calculations}

\section{One-term homology}

The work done in previous sections demonstrates that one-term homology for shelves with a left zero or a right unit is trivial for every positive dimension. The first three one-term homology groups for some associative shelves of size four not satisfying these conditions appear below (Tables 11, 12, 13, 14):

The one-term homology groups are:

(1) $H_{0}^{(*)}\left(T_{1}\right)=\mathbb{Z}, \quad H_{1}^{(*)}\left(T_{1}\right)=0, \quad H_{2}^{(*)}\left(T_{1}\right)=0$

(2) $H_{0}^{(*)}\left(T_{2}\right)=\mathbb{Z}^{2}, \quad H_{1}^{(*)}\left(T_{2}\right)=\mathbb{Z}^{4}, \quad H_{2}^{(*)}\left(T_{2}\right)=\mathbb{Z}^{16}$

(3) $H_{0}^{(*)}\left(T_{3}\right)=\mathbb{Z}^{3}, \quad H_{1}^{(*)}\left(T_{3}\right)=\mathbb{Z}^{8}, \quad H_{2}^{(*)}\left(T_{3}\right)=\mathbb{Z}^{32}$

(4) $H_{0}^{(*)}\left(T_{4}\right)=\mathbb{Z}^{4}, \quad H_{1}^{(*)}\left(T_{4}\right)=\mathbb{Z}^{12}, \quad H_{2}^{(*)}\left(T_{4}\right)=\mathbb{Z}^{48}$

Table $11 T_{1}$

\begin{tabular}{l|llll}
$*$ & 0 & 1 & 2 & 3 \\
\hline 0 & 0 & 0 & 0 & 3 \\
1 & 0 & 0 & 0 & 3 \\
2 & 0 & 0 & 2 & 3 \\
3 & 0 & 0 & 3 & 3
\end{tabular}

Table $12 T_{2}$

\begin{tabular}{l|llll}
$*$ & 0 & 1 & 2 & 3 \\
\hline 0 & 0 & 0 & 2 & 2 \\
1 & 1 & 1 & 3 & 3 \\
2 & 0 & 0 & 2 & 2 \\
3 & 1 & 1 & 3 & 3
\end{tabular}

Table $13 \quad T_{3}$

\begin{tabular}{l|llll}
$*$ & 0 & 1 & 2 & 3 \\
\hline 0 & 0 & 0 & 2 & 3 \\
1 & 0 & 1 & 2 & 3 \\
2 & 0 & 0 & 2 & 3 \\
3 & 0 & 0 & 2 & 3
\end{tabular}


Table $14 T_{4}$

\begin{tabular}{l|llll}
$*$ & 0 & 1 & 2 & 3 \\
\hline 0 & 0 & 1 & 2 & 3 \\
1 & 0 & 1 & 2 & 3 \\
2 & 0 & 1 & 2 & 3 \\
3 & 0 & 1 & 2 & 3
\end{tabular}

\section{Two-term homology}

Our main result, Theorem 3.3, completely describes the two-term homology groups of shelves with right fixed elements. However, there exist associative shelves without right fixed elements. The first three two-term homology groups for some associative shelves of size four without right fixed elements, together with the first three homology groups of the unital shelf (15), appear below (Tables 15, 16, 17, 18):

Table $15 T_{5}$

\begin{tabular}{l|llll}
$*$ & 0 & 1 & 2 & 3 \\
\hline 0 & 0 & 0 & 0 & 0 \\
1 & 0 & 1 & 1 & 1 \\
2 & 0 & 1 & 2 & 2 \\
3 & 0 & 1 & 2 & 3
\end{tabular}

Table $16 T_{6}$

\begin{tabular}{l|llll}
$*$ & 0 & 1 & 2 & 3 \\
\hline 0 & 0 & 0 & 0 & 0 \\
1 & 0 & 0 & 0 & 0 \\
2 & 0 & 0 & 0 & 0 \\
3 & 3 & 3 & 3 & 3
\end{tabular}

Table $17 \quad T_{7}$

\begin{tabular}{l|llll}
$*$ & 0 & 1 & 2 & 3 \\
\hline 0 & 0 & 0 & 0 & 0 \\
1 & 0 & 0 & 0 & 0 \\
2 & 2 & 2 & 2 & 2 \\
3 & 3 & 3 & 3 & 3
\end{tabular}


Table $18 T_{8}$

\begin{tabular}{l|llll}
$*$ & 0 & 1 & 2 & 3 \\
\hline 0 & 0 & 0 & 0 & 0 \\
1 & 1 & 1 & 1 & 1 \\
2 & 2 & 2 & 2 & 2 \\
3 & 3 & 3 & 3 & 3
\end{tabular}

The two-term homology groups are:

$\begin{array}{lll}\text { (1) } H_{0}^{R}\left(T_{5}\right)=\mathbb{Z}, & H_{1}^{R}\left(T_{5}\right)=\mathbb{Z}, & H_{2}^{R}\left(T_{5}\right)=\mathbb{Z} \\ \text { (2) } H_{0}^{R}\left(T_{6}\right)=\mathbb{Z}^{2}, & H_{1}^{R}\left(T_{6}\right)=\mathbb{Z}^{4}, & H_{2}^{R}\left(T_{6}\right)=\mathbb{Z}^{8} \\ \text { (3) } H_{0}^{R}\left(T_{7}\right)=\mathbb{Z}^{3}, & H_{1}^{R}\left(T_{7}\right)=\mathbb{Z}^{9}, & H_{2}^{R}\left(T_{7}\right)=\mathbb{Z}^{27} \\ \text { (4) } H_{0}^{R}\left(T_{8}\right)=\mathbb{Z}^{4}, & H_{1}^{R}\left(T_{8}\right)=\mathbb{Z}^{16}, H_{2}^{R}\left(T_{8}\right)=\mathbb{Z}^{64}\end{array}$

It is not difficult to notice the pattern suggested from the data above, and thus the results for all other associative shelves of size four are not included. This pattern holds for racks $[6,17]$. Additional computations of torsion in two-term homology for quandles were performed in $[19,25]$ for example.

\section{References}

1. Biondi, E., Divieti, L., Guardabassi, G.: Counting paths, circuits, chains, and cycles in graphs: a unified approach. Can. J. Math. 22, 22-35 (1970)

2. Burstin, C., Mayer, W.: Distributive gruppen endlicher ordnung (english translation: Finite distributive groups). J. Reine Angew. Math 160, 111-130 (1929). doi:10.1515/crll.1929.160.111. (German). arXiv: 1403.6326

3. Crans, A., Przytycki, J.H., Putyra, K.K.: Torsion in one term distributive homology. Fundamenta Mathematicae, vol. 225, pp. 75-94 (2014). arXiv:1306.1506

4. Dehornoy, P.: Progress in Mathematics. Braids and self-distributivity, vol. 192. Birkhäuser, Basel (2000)

5. Dehornoy, P., Lebed, V.: Two- and three-cocycles for Laver tables. J. Knot Theory Ramif. 23(4), 1450017 (2014)

6. Etingof, P., Graña, M.: On rack cohomology. J. Pure Appl. Algebra 177, 49-59 (2003). arXiv:math/0201290v2 [math.QA]

7. Fenn, R., Rourke, C., Sanderson, B.: An introduction to species and the rack space. Bozhuyuk, M.E. (ed.), Topics in Knot Theory. Proceedings of the Topology Conference, Erzurum, NATO Adv. Sci. Inst. Ser. C. Math. Phys. Sci., vol. 399, pp. 33-35. Kluwer Academic Publishers, Amsterdam (1993)

8. Fenn, R., Rourke, C., Sanderson, B.: Trunks and classifying spaces. Appl. Categ. Struct. 3, 321-356 (1995)

9. Fenn, R., Rourke, C., Sanderson, B.: James Bundles and Applications (1996). http://www.maths. warwick.ac.uk/cpr/ftp/james.ps. (preprint)

10. Guardabassi, G.: Counting constrained routes in complete networks: the H-function. (unpublished notes cited in [BDG])

11. Ježek, J., Kepka, T.: Selfdistributive groupoids Part D1: left distributive semigroups. Acta Universitatis Carolinae. Mathematica et Physica 47(1), 15-56 (2006). https://eudml.org/doc/246241 ?lang=es\& limit $=15$

12. Kepka, T.: Varieties of left distributive semigroups. Acta Univ. Carolinae Math. Phys. 22(2), 23-37 (1981)

13. Kimura, N.: The structure of idempotent semigroups I. Pac. J. Math. 8(2), 257-275 (1958)

14. Laver, R.: On the algebra of elementary embeddings of a rank into itself. Adv. Math. 110(2), 334-346 (1995). arXiv:math/9204204v1 [math.LO] 
15. Lawvere, F.W.: Qualitative distinctions between some toposes of generalized graphs. Contemp. Math. 92, 261-299 (1989)

16. Lebed, V.: Cohomology of finite monogenic self-distributive structures. J. Pure Appl. Algebra 220(2), 711-734 2016. arXiv:1503.07030

17. Litherland, R.A., Nelson, S.: The Betti numbers of some finite racks. J. Pure Appl. Algebra 178, 187-202 (2003). arXiv:math/0106165v4 [math.GT]

18. Loday, J.L.: Cyclic homology. In: Grund. Math. Wissen. Band, vol. 301. Springer, Berlin (1992). (second edition, 1998)

19. Niebrzydowski, M., Przytycki, J.H.: Homology of dihedral quandles. J. Pure Appl. Algebra 213, 742755 (2009). http://front.math.ucdavis.edu/math.GT/0611803

20. Peirce, C.S.: On the algebra of logic. Am. J. Math. 3(1), 15-57 (1880)

21. Petrich, M.: Introduction to Semigroups. Charles E.Merrill Publishing Company, Columbus (1973)

22. Przytycki, J.H.: Distributivity versus associativity in the homology theory of algebraic structures. Demonstratio Mathematica 44(4) (2011). arXiv:1109.4850

23. Przytycki, J.H.: Knots and distributive homology: from arc colorings to Yang-Baxter homology. In: New Ideas in Low Dimensional Topology, vol. 56, pp. 413-488. World Scientific, Singapore (2015). arXiv: 1409.7044 [math.GT]

24. Przytycki, J.H., Sikora, A.S.: Distributive products and their homology. Commun. Algebra 42(3), 1258-1269 (2014). arXiv:1105.3700

25. Przytycki, J.H., Yang, S.Y.: The torsion of a finite quasigroup quandle is annihilated by its order. J. Pure Appl. Algebra 219(10), 4782-4791 (2015). arXiv:1411.1727

26. Sam, C.: Blog of John Baez, May 23, 2015

27. Schröder, E.: Über Algorithmen und Calculn. Archive der Math. und Phys. 5, 225-278 (1887)

28. Shützenberger, M.P.: Sur certains treillisgauches. C. R. Acad. Sci. Paris 225, 277-278 (1947)

29. Zejnullahu, A.: Free left distributive semigroups. Acta Universitatis Carolinae. Mathematica et. Physica 30(1), 29-32 (1989) 